\title{
Effectiveness and Safety of the European Society of Cardiology 0-/1-h Troponin Rule-Out Protocol: The Design of the ESC-TROP Multicenter Implementation Study
}

\author{
Arash Mokhtari $^{\mathrm{a}}$ Ardavan Khoshnood $^{\mathrm{b}}$ Jakob Lundager Forberg ${ }^{\mathrm{c}}$ \\ Caroline Hård af Segerstad ${ }^{d}$ Ulf Ekström ${ }^{e}$ Tommy Schyman $^{f}$ \\ Mahin Akbarzadeh ${ }^{\mathrm{b}}$ Bertil Lindahlg Ulf Ekelund $^{\mathrm{b}}$

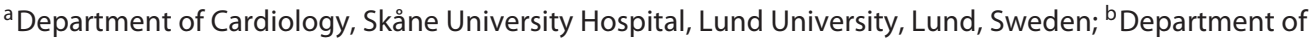 \\ Emergency Medicine, Skåne University Hospital, Lund University, Lund, Sweden; ' Department of Emergency \\ Medicine, Helsingborg General Hospital, Helsingborg, Sweden; ${ }^{d}$ Department of Emergency Medicine,

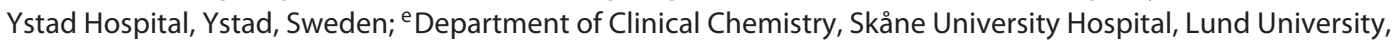 \\ Lund, Sweden; ${ }^{\mathrm{f} C l i n i c a l}$ Studies Sweden, Forum South, Skåne University Hospital, Lund, Sweden; \\ ${ }^{9}$ Department of Medical Sciences, Clinical Research Center, Uppsala University, Uppsala, Sweden
}

\section{Keywords}

Acute coronary syndrome $\cdot$ Chest pain · Myocardial infarction · High-sensitivity cardiac troponin · Emergency department

\begin{abstract}
Chest pain is one of the most common complaints at the emergency department (ED), and it is commonly the perceived likelihood of acute coronary syndrome (ACS) that drives management. Guidelines from the European Society of Cardiology (ESC) recommend the use of a 0-/1-h high-sensitivity cardiac troponin $\mathrm{T}$ (hs-cTnT) protocol to rule out or in ACS, but this is mostly based on observational studies. The aim of the ESC-TROP trial is to determine the safety and effectiveness of the ESC 0-/1-h hs-cTnT protocol when implemented in routine care. Adult chest pain patients at 5 EDs in the Skåne Region, Sweden, are included in the trial. The 0-/ 1-h hs-cTnT ESC protocol supplemented with clinical assessment and electrocardiography (ECG) is implemented at 3
\end{abstract}

EDs, and the other 2 EDs act as concurrent controls. Outcomes will be evaluated during the 10 months after the implementation and the corresponding 10 months of the previous year. The 2 co-primary outcomes are (a) acute myocardial infarction (AMI) and all-cause death within 30 days in patients discharged from the ED, and (b) ED length of stay of the same patients. Secondary outcomes include the proportion of chest pain patients discharged from the ED and the number of ruled-out patients undergoing objective testing within 30 days. The ESC-TROP trial will determine the performance and applicability of the 0-/1-h hs-cTnT ESC protocol supplemented with clinical assessment and ECG when implemented in routine ED care. It will provide evidence whether 0-/1-h hs-cTnT testing is safe, effective, and feasible, and whether widespread implementation as recommended by ESC guidelines should be supported.

C 2020 The Author(s)

Published by S. Karger AG, Basel

Trial Registration: NCT03421873, registered on February 5, 2018 (https://clinicaltrials.gov/ct2/show/NCT03421873).
(C) 2020 The Author(s)

Published by S. Karger AG, Basel

This is an Open Access article licensed under the Creative Commons Attribution-NonCommercial-4.0 International License (CC BY-NC) (http://www.karger.com/Services/OpenAccessLicense), applicable to the online version of the article only. Usage and distribution for commercial purposes requires written permission.
Arash Mokhtari

Department of Cardiology

Skåne University Hospital, Lund University

Klinikgatan 15, SE-221 85 Lund (Sweden)

arash.mokhtari.0561@med.lu.se 


\section{Introduction}

Chest pain is a common complaint at the emergency department (ED), with 15-20 million yearly visits in Europe and the US. The list of potential diagnoses is long, but it is mainly the risk of acute coronary syndrome (ACS), i.e., acute myocardial infarction (AMI) or unstable angina (UA), that drives patient management. The fear of missing patients with ACS leads to lengthy assessments in the ED and high admission rates [1], which contribute to ED and hospital crowding and a decreased quality of care [2]. However, only $10-15 \%$ of all ED chest pain patients and $<25 \%$ of admitted chest pain patients prove to have ACS [3]. Many of the admissions and investigations are thereby unnecessary and cause a substantial health care burden [1].

The ED assessment of possible ACS is based on history, physical examination, electrocardiography (ECG), and cardiac troponin measurements [4]. In this context, a number of observational studies have indicated that high-sensitivity cardiac troponin (hs-cTnT) testing at patient presentation $(0 \mathrm{~h})$ and $1 \mathrm{~h}$ later $(1 \mathrm{~h})$ can safely rule out AMI [5-7]. Based on these studies, the European Society of Cardiology (ESC) guidelines strongly recommend (class 1) the use of the 0-/1-h hs-cTnT protocol [8].

A large proportion of patients undergo further stress testing/cardiac imaging or coronary angiography (below referred to as objective testing) [9] in order to also exclude $\mathrm{UA}$, and this approach is recommended by guidelines in patients with a negative ECG and blood biomarkers [10]. However, neither routine admission nor objective testing has been shown to improve outcome in low-risk chest pain patients $[9,11,12]$, and may in fact be more harmful than beneficial $[11,12]$.

There are therefore several knowledge gaps, which this study aims to address. First, all studies underlying the ESC protocol before the start of this study in 2017 had been strictly observational, with no patients managed according to the protocol. Four studies on the implementation of a $0-/ 1-h$ protocol have been published since then, but they have all been smaller than the present study, they have had somewhat diverging results, and one of them lacked a control arm [13-16]. Second, although the ESC guidelines state that the protocol should be combined with ECG and patient history, they neither specify how this should be done, nor the expected effect on safety and efficacy. This was also not addressed in the recent implementation studies. Third, the ESC protocol does not specify how to identify the low risk patients who should undergo further objective testing in order to exclude UA.
Our previous studies indicate that $0-/ 1-\mathrm{h}$ hs-cTnT combined with clinical risk stratification can identify patients with such a low risk of 30-day MACE including UA [5, 6, 17] that neither admission nor further cardiac testing is needed. Thus, there is a clear need for further studies on the performance of $0-/ 1-h$ hs-cTnT testing in routine care.

The aim of this trial is to determine the safety and effectiveness of the ESC 0-/1-h hs-cTnT protocol, supplemented with clinical assessment and ECG, when implemented in routine care, including a recommendation to refrain from further objective testing in low-risk patients.

\section{Methods and Design}

\section{Setting}

Patients are enrolled at all 5 EDs open 24/7 in the Skåne Region, the southernmost county in Sweden, since all hospitals in our region use the same electronic patient record system which allows good data collection and follow-up, and since all use hscTnT analyzed on Cobas instruments from Roche Diagnostics (Basel, Switzerland). The protocol is implemented in 3 of these EDs; 1 tertiary-care university ED (Lund, 5,500 chest pain patients yearly), 1 large community ED (Helsingborg, 5,000 chest pain patients yearly), and 1 rural community ED (Ystad, 2,000 chest pain patients yearly). The remaining EDs are 1 university ED (Malmö, 7,000 chest pain patients yearly) and 1 community ED (Kristianstad, 3,500 chest pain patients yearly), and they will not implement the protocol but act as concurrent controls. At the 5 participating EDs, only patients $\geq 18$ years old are evaluated. Patients with ST-elevation myocardial infarction are identified in the ambulance and brought directly to the cardiac catheterization laboratory, bypassing the ED.

\section{Study Design}

ESC-TROP is a pragmatic before-and-after study with concurrent controls evaluating the implementation of a diagnostic algorithm. The intervention group consists of ED chest pain patients enrolled after implementation of the $0-/ 1-\mathrm{h}$ hs-cTnT protocol at the 3 intervention EDs, and this period constitutes the "after period." Comparisons will then be made with patients seeking care at the intervention EDs during the corresponding 10 months of the previous year, which constitutes the "before period" (i.e., before implementation of the 0 -/1-h hs-cTnT protocol). Differences between the before-and-after period in the intervention EDs will then be compared with the differences between the before-andafter period at the 2 control hospitals. The study design is outlined in online supplementary Appendix 1 (for all online suppl. material, see www.karger.com/doi/10.1159/000509390). The study enrollment started on February 1, 2018, and the inclusion has now been finished. The run-in period did not lead to any adjustments before the implementation period.

\section{Patient Inclusion and Informed Consent}

The electronic ED patient log is assessed for ED patients with a primary complaint of nontraumatic chest pain who are then 
Table 1. Exclusion criteria

- A final diagnosis of STEMI during the index visit, as this diagnosis is not based on blood biomarkers

- No hs-cTnT ordered, as this signifies no suspicion of acute coronary syndrome

- Leaving the ED against medical advice

- No Swedish personal identity number, because those patients cannot be followed up via national registries

- Previous enrollment: patients will only be enrolled during their first ED visit

- Patients who actively decline participation; this study has received ethical approval without the need for written informed consent; information will however be posted in the EDs informing patients that data will be collected from the electronic health records and registries, and that they can withdraw from participation at any time, without specific reason by contacting the study administration

ED, emergency department; hs-cTnT, high-sensitivity cardiac troponin T; STEMI, ST-elevation myocardial infarction.

Table 2. Primary and secondary outcomes

Primary outcomes

1. The rate of AMI and all-cause death within 30 days from ED presentation in patients discharged from the ED (safety)

2. ED length of stay in patients discharged from the ED (effectiveness)

Key secondary outcomes

1. Proportion of patients discharged from the ED

2. Proportion of patients fulfilling $0-/ 1-\mathrm{h}$ hs-cTnT criteria who undergo objective testing within 30 days during the intervention period (no comparison with before intervention)

3. Proportion of patients without acute coronary syndrome admitted to the coronary care unit

4. Proportion of patients who undergo inappropriate coronary angiographies

5. Total hospital length of stay

6. Proportion of patients with 30 -day ED revisits

7. Proportion of discharged patients with admissions to inpatient care within 30 days

8. Health care cost within 30 days

AMI, acute myocardial infarction; ED, emergency department; hs-cTnT, high-sensitivity cardiac troponin T.

screened for eligibility. A flow diagram is presented in online supplementary Appendix 2. All chest pain patients are thus enrolled by default. The exclusion criteria are presented in Table 1.

\section{Study Intervention: The Implemented Chest Pain Protocol}

The 0-/1-h hs-cTnT protocol (online suppl. Appendix 3) used in this study is based on the protocol proposed in the ESC guidelines, with the addition of clinical assessment and ECG. The protocol reflects real-life practice where management is not based solely on the hs-cTnT level, and it has been shown to outperform a protocol based on hs-cTnT levels alone [5]. In addition, the protocol provides clear suggestions for management. A 1-h hs-cTnT is defined as a second hs-cTnT sample drawn 45-90 min after the first sample drawn on admission (0 h) [7].

Before starting the outcome data collection, there is a 2-month run-in period for protocol implementation. ED physicians, internal medicine physicians, cardiologists, and ED nurses in Lund, Helsingborg, and Ystad are given a standard lecture and pocket cards detailing the management recommended by the protocol. Posters and written guidance on the use of the protocol are also distributed in the ED, the telemetry unit, and the cardiology department.

According to the protocol, patients are stratified into different risk categories for ACS: low, intermediate, and high risk.

Patients are classified at low risk if they meet all the following criteria:

- The physician does not assess the patient's risk of ACS as high based on the history and physical examination (online suppl. Appendix 3).

- The physician does not consider the ECG to show signs of acute ischemia (online suppl. Appendix 3).

- The 0 -h hs-cTnT is $<5 \mathrm{ng} / \mathrm{L}$ or the 0 -h hs-cTnT is $<12 \mathrm{ng} / \mathrm{L}$ with a 0 - to 1 -h increase $<3 \mathrm{ng} / \mathrm{L}$.

In these patients, physicians are recommended to refrain from further cardiac testing. They are instead encouraged to consider alternative diagnoses, and to recommend patients at discharge to seek follow-up if their symptoms have not improved within 7 days and to return to the ED in case symptoms progress. We chose an increase instead of delta in this setting, as this was felt to be clinically sensible and in line with guideline recommendations $[8,18]$. 
Due to the lack of data regarding $0-\mathrm{h} / 1-\mathrm{h}$ testing in early presenters, i.e., patients with a 0 -h hs-cTnT test $\leq 2 \mathrm{~h}$ from symptom onset [19], these patients are recommended to undergo a 2-h hscTnT test.

Patients are classified at high risk if they meet at least one of the following criteria:

- 0 -h hs-cTnT $\geq 52 \mathrm{ng} / \mathrm{L}$.

- 0 - to 1 -h hs-cTnT change $\geq 5 \mathrm{ng} / \mathrm{L}$.

- The physician's clinical assessment, based on history and physical examination, is that the risk of ACS is high AND the hs-cTnT is $>14 \mathrm{ng} / \mathrm{L}$.

- History indicating crescendo angina or new-onset angina.

- New or presumed new ischemic ECG changes.

In these patients, the cardiology consultant should be contacted, and the patient preferably admitted.

The remaining patients are considered at intermediate risk. Physicians are recommended to contemplate important differential diagnoses and order an additional hs-cTnT after 3-6 h in patients with a remaining suspicion of ACS, as well as to consider further diagnostic testing such as myocardial perfusion imaging, CT coronary angiography, and/or echocardiography.

\section{Control Group}

The control arm will consist of both chest pain patients managed at the 3 intervention EDs during the corresponding 10 months of the previous year (intervention hospitals acting as their own controls) as well as chest pain patients managed during the corresponding before-and-after period at EDs not implementing the protocol (concurrent controls).

The standard of care before the intervention period and in the control hospitals has been the use of 0 - and 3-h hs-cTnT, which has been recommended by the ESC since 2011 [20]. In accordance with the guidelines, patients are usually considered at low risk for AMI if they have a 0 - and 3-h hs-cTnT $\leq 14 \mathrm{ng} / \mathrm{L}$, or a hs-cTnT on admission $\leq 14 \mathrm{ng} / \mathrm{L}$ measured $>6 \mathrm{~h}$ after symptom onset [21].

\section{Primary Outcomes}

The following outcomes will be compared in the 10-month periods before and after the implementation, and for differences in change between intervention and control hospitals (Table 2):

(1) The Incidence of AMI and All-Cause Death within 30 Days from ED Presentation (Not Including the Index Visit) in Patients Discharged from the ED. This outcome will evaluate the safety of the $0-/ 1$-h hs-cTnT protocol in a noninferiority analysis based primarily on a comparison of the before and after period in the intervention hospitals, and we will also compare the differences in change between intervention and control hospitals.

Deaths and dates of death will be retrieved from the Swedish population register and data on AMIs and hospital visits from the SWEDEHEART quality register and the Swedish national patient register. These registries have excellent coverage and accuracy [22, 23 ], and the approach will provide close to $100 \%$ nationwide coverage of AMI and death events.

All discharged patients with a possible event within 30 days will have their event reviewed and adjudicated by 2 independent cardiologists blinded to the index visit assessment $(0 / 1$ or $0 / 3 \mathrm{~h})$ and in case of disagreement by the adjudication committee. AMI is defined according to the universal definition requiring a significant rise and/or fall in hs-cTnT with $\geq 1$ value above the 99th percentile, combined with symptoms or signs of cardiac ischemia
[24]. Significant hs-cTnT change is defined as an absolute change $\geq 5 \mathrm{ng} / \mathrm{L}$ within $1 \mathrm{~h}$ or $>7 \mathrm{ng} / \mathrm{L}$ within $2-3 \mathrm{~h}$, or $\geq 9 \mathrm{ng} / \mathrm{L}$ within $6 \mathrm{~h}$, and/or a change $>20 \%$ if the 0 -h hs-cTnT is $>14 \mathrm{ng} / \mathrm{L}$ [25]. To avoid misclassification of patients presenting in a troponin plateau phase, an AMI diagnosis can still be adjudicated in patients with elevated hs-cTnT levels in the absence of a significant rise or fall if AMI is considered to be the most likely diagnosis based on all available information [24].

All-cause death will be used in the primary outcome, but the proportion of cardiac deaths will also be adjudicated. Cardiac death will be defined as sudden cardiac death and death caused by ACS or other coronary artery disease, congestive heart failure, or cardiac arrhythmia, all according to standardized data definitions for ACS research [26]. We will also analyze how many missed cases of $\mathrm{AMI} /$ death that were managed in accordance with the $0-/ 1$-h protocol, and the number of protocol violations.

(2) The ED Length of Stay (LOS) in Patients Discharged from the ED. This outcome evaluates the effectiveness of the protocol to reduce ED LOS in discharged patients. These data will be obtained from the electronic ED patient log and medical records at each ED.

\section{Secondary Outcomes}

The following secondary outcomes will also be compared before and after the implementation at all 5 EDs.

1. Proportion of Patients Discharged from the ED. This will evaluate the effectiveness of the protocol, and data will be obtained similarly to ED LOS as stated above.

2. Proportion of Patients Fulfilling 0-/1-h hs-cTnT Rule-Out Criteria Who Undergo Objective Testing within 30 Days during the Intervention Period (No Comparison with before Intervention). This will describe physician compliance with the protocol, and whether this is safe. Data on objective testing (exercise stress test, myocardial perfusion imaging, CT coronary angiography, cardiac magnetic resonance perfusion imaging, or coronary angiography, including results) within 30 days from the ED visit will be retrieved from electronic medical records from the entire Skåne Region, including primary care. Referrals for objective testing will be categorized as appropriate, inappropriate/protocol violation, or unclear. Data will be provided on the incidence of AMI and all-cause death within 30 days among those fulfilling $0-/ 1-\mathrm{h}$ hs-cTnT rule-out criteria where objective testing is not performed. Among those referred for further testing, data will be provided regarding the proportion of positive tests, and how many of these were true positives, and how many were false positives, as well as the proportion of tests performed in an out- versus inpatient setting.

3. Proportion of Non-ACS Patients Admitted to the Coronary Care Unit (CCU). This is to evaluate whether the $0-/ 1-\mathrm{h}$ protocol will increase admission of patients without ACS to the CCU due to the rule-in criteria being too unspecific. Data on ACS diagnoses and CCU admissions will be obtained from the SWEDEHEART register and electronic medical records.

4. Proportion of Patients Who Undergo Inappropriate Coronary Angiographies. This is also to evaluate whether the rule-in criteria are to unspecific and lead to an increase in inappropriate angiographies. Data on coronary angiography and ACS diagnoses will be obtained from SWEDEHEART, where the Swedish Coronary Angiography and Angioplasty Register (SCAAR) has $100 \%$ nationwide coverage [22]. 
5. Total Hospital LOS. Time from ED presentation to hospital or ED discharge, obtained from electronic medical records. This will also be analyzed separately for the subgroup without ACS as discharge diagnosis.

6. Proportion of Patients with 30-Day ED Revisits. This evaluates whether the implementation of the protocol, via increasing the discharge of low-risk patients without further objective testing, results in a change in ED revisits. Data will be obtained from the electronic ED patient log, patient records, and the national patient register.

7. Proportion of Discharged Patients with Admission to Inpatient Care within 30 Days. Data will be obtained from the electronic patient records and the national patient register.

8. Health Care Costs within 30 Days. Data on the direct health care cost of each patient will be obtained from the administrative systems of the Skåne Region.

\section{Retrieval of Additional Patient Data and Follow-Up}

Data on patient characteristics, including previous diseases, current medications, results of blood tests, ED management, admission to inhospital care, and outpatient follow-up will be collected from the electronic ED patient logs, the regional electronic medical record systems, hospital laboratories, and national registries (the Swedish emergency medicine quality register (ANSWER), the Swedish national patient register, the Swedish population register, the Swedish prescribed drug register, and SWEDEHEART) [23, 27].

As data from comprehensive regional patient records as well as national registries on all patients are available, only migrators to other countries within 30 days will be lost to follow-up, and they are expected to be few. There will be no telephone follow-up since this is a pragmatic implementation study and since knowing patients will have telephone follow-up may falsely inflate the discharge rate. Follow-up by extensive electronic medical records has also been shown to be as accurate as by telephone [28].

\section{Data Analyses}

All data management will be in accordance with applicable Swedish laws and performed by Clinical Studies Sweden Forum South, an independent organization, who will also perform the statistical analyses. All patients meeting the inclusion criteria and not meeting exclusion criteria will be included in the analyses, regardless of compliance with the protocol (intention to treat). This will reflect the true safety and effectiveness of the protocol in routine care where full compliance will not be attained, and misinterpretations will occur.

\section{Analyses of Endpoints}

When comparing the outcomes before and after the intervention, a hierarchical testing procedure will be used:

Step 1. Event rate (30-day AMI/death) after implementation (intervention EDs) will be compared to that in the control period using a noninferiority approach (see Sample Size, below).

Step 2. If the event rate after implementation is not inferior to before, primary effectiveness and secondary outcomes will be analyzed. Testing will also be performed to assess for superiority in the safety outcome.

Step 1 will be analyzed using Newcombe 95\% CI for the difference between the proportions, calculated as event rate in the intervention group minus event rate in the control group. If the upper limit of this confidence interval is below the noninferiority margin, the conclusion will be that the event rate in the intervention group is noninferior to the event rate in the control group, and analyses in step 2 will be performed.

For the primary effectiveness outcome, ED LOS, a generalized linear mixed model will be used, considering potential confounders and adjusting for potential cluster effects between EDs. Also, interrupted time series analysis will be used to adjust the potential intervention effect for underlying time trends. For the secondary outcomes, a generalized linear mixed model, Pearson $\chi^{2}$, Fisher's exact test, $t$ test, or Mann-Whitney $\mathrm{U}$ will be used, as appropriate. The difference in change between the intervention and control hospitals will be evaluated using a generalized linear model. All $p$ values will be 2 -sided, and a $p$ value $<0.05$ will be considered significant.

\section{Additional Analyses}

The primary outcomes will also be analyzed for chest pain patients at the 3 enrolling EDs during the 2 years preceding the 10 -month pre-implementation period to evaluate for secular trends in chest pain management. Further, we will analyze ED LOS before and after the implementation in patients with a chief complaint of dyspnea or abdominal pain at the same EDs, to evaluate for temporal trends in general patient management.

In a subset of patients, the assessment (low, intermediate, or high risk) will be made by 2 independent ED physicians to analyze the degree of agreement in the interpretation of the protocol.

Data regarding physician satisfaction with patient management will be collected through an electronic survey to all ED physicians before and after the implementation of the protocol.

Detailed data on all missed cases as well as outcomes for patients with a 0 -h hs-cTnT $<12 \mathrm{ng} / \mathrm{L}$ and a decrease $\geq 3 \mathrm{ng} / \mathrm{L}$ will be provided.

Subgroup analyses are planned for both primary outcomes in the following subgroups: enrolling EDs, men and women, age $<65$ and $\geq 65$ years, presentation during dayshift versus nightshift, history of coronary artery disease versus no history, history of diabetes versus no history, estimated glomerular filtration rate $<60$ versus $>60 \mathrm{~mL} / \mathrm{min} / 1.73 \mathrm{~m}^{2}$, and $\leq 2$ versus $>2$ h from ED admission to first physician contact.

\section{Sample Size}

Assuming an event rate (30-day AMI/death) of $0.4 \%$ among discharged patients in the control group [29] and an event rate of $0.5 \%$ after the implementation, a total of 8,792 discharged patients would be needed to determine that the event rate in the intervention group is noninferior to that in the control group, with an a risk of 0.05 and an $80 \%$ power. The noninferiority margin is set to $0.5 \%$ units. Based on this and about 13,100 annual chest pain visits in the intervention EDs, a discharge rate of 55\%, an exclusion rate of $25 \%$, and some safety margin, our goal is to include 8,200 patients in the intervention hospitals during each 10 -month period. With this sample size and with an estimated median ED LOS in discharged patients in the control group of $240 \mathrm{~min}$, we will be able to detect a 14 -min difference in LOS with $80 \%$ power and 0.05 a risk. This sample size will thereby provide adequate power to detect a clinically significant difference in ED LOS for the co-primary outcome.

\section{Feasibility and Study Progress}

The main results (primary outcome) of the ESC-TROP trial will be available in 2020, and results for the secondary outcomes in 2020 and 2021. 
Strengths and Limitations

ESC-TROP is a pragmatic before-and-after implementation study with concurrent controls, and as such it will provide information on the true effectiveness and safety of the $0-/ 1-h$ hs-cTnT protocol in routine care. The different sizes and settings at the participating EDs, as well as the by-default inclusion of all chest pain patients, will probably make the results highly generalizable. The default inclusion will also eliminate the risk of selection bias.

Previous studies on the 0 -/1-h hs-cTnT protocol have primarily included patients with chest pain only $[6,30]$, and the performance in patients with other presenting symptoms such as dyspnea or syncope, where the pretest probability for ACS is lower, is therefore uncertain. We have therefore chosen to evaluate the effects of implementing the protocol in chest pain patients only. The results and conclusions of the ESC-TROP study will only be applicable to patients presenting with chest pain. Patients with type 2 AMI often present with other primary complaints than chest pain. As such, we will not be able to evaluate the performance of this algorithm for type 2 AMI other than in the setting of a primary complaint of chest pain.

Non-Swedish citizens are excluded from the study since these patients will be missed in the register-based follow-up. The number of non-Swedish citizens at the participating EDs is likely very small, and we believe that their exclusion will have a negligible effect on the generalizability of the results.

At the participating EDs in the present study, standard of care has been to order a hs-cTnT at presentation and $3 \mathrm{~h}$ later. Recent studies have however shown that the 3-h hs-cTnT approach may lead to an unacceptably high miss rate $[31,32]$. A $0-/ 1 \mathrm{~h}$ protocol has consistently been shown to be safe in several multicenter observational studies and is recommended (class 1) by ESC guidelines. We therefore believe that the possible benefits of implementing this protocol clearly outweighs any potential risks, and that the present study will provide important information for others who consider implementing the protocol. Ultimately, treatment and disposition decisions are at the discretion of the physicians who may choose to override the protocol recommendations if they believe it to be in the best interest of the patient.

The study information - including being enrolled by default/ without consent - is given as posters in several highly visible places in the EDs, and as leaflets distributed to all patients requesting them. Still, we cannot exclude that some patients miss the information. The information and opt-out procedures in the study have been approved by the Regional ethics review board.

\section{Discussion}

ED patients with acute chest pain are common, and the fear of missing ACS leads to lengthy assessments and many admissions to inhospital care, which contributes to ED and hospital crowding. In the end, only a small fraction of these patients proves to have ACS, and these unnecessary investigations and admissions consume large health care resources. There is therefore a strong need for improved methods in the assessment of ED chest pain patients.
The now commonly used hs-cTnT has an improved analytical and diagnostic sensitivity compared to previous-generation cTnT, and it enables faster exclusion of AMI and reductions in ED LOS and costs $[30,33]$. Their introduction has also resulted in a decrease in the proportion of patients diagnosed with UA [33], further diminishing the potential gain of objective testing in those identified as low risk. However, many clinicians are uncertain as how to apply hs-cTnT in practice, and many hospitals lack hs-cTnT protocols to guide physicians, although the unstructured use of hs-cTnT in ED chest pain patients does not confer a significant benefit [34]. There is a clear need for protocols that can provide a framework for optimal use, and this is supported by international guidelines with a class $1 \mathrm{~A}$ level of recommendation [35].

The ESC-TROP trial is the first to analyze the performance and applicability of the ESC recommended 0-1 1-h hs-cTnT protocol in routine care, supplemented with clinical assessment and ECG. Even though the 0 -/1-h hs-cTnT protocol has been validated in different settings, prior studies have primarily been observational, and patients have not been managed in accordance with the protocol $[5,17]$. Since the true safety and effectiveness of the protocol in routine care is thereby unknown, many physicians have hesitated to implement the protocol in routine care. Additionally, this study evaluates an approach where we recommend discharging low-risk patients without further objective testing, an approach which has not previously been evaluated in a large prospective study. The trial will thereby fill an important knowledge gap.

If the implemented hs-cTnT protocol can rapidly identify a large proportion of chest pain patients suitable for early discharge with no need for further cardiac testing, this may reduce ED and hospital crowding, objective testing, and health care costs, and will benefit both patients and the health care system.

\section{Conclusion}

The ESC-TROP trial will determine the performance and applicability of the 0-/1-h hs-cTnT ESC protocol, supplemented with clinical assessment and ECG, when implemented in routine ED care. It will provide evidence whether 0-/1-h hs-cTnT testing is safe, effective, and feasible, and whether widespread implementation as recommended by guidelines should be supported. 


\section{Statement of Ethics}

The information and opt-out procedures in the study have been approved by the regional ethics review boards. This study has received ethical approval without the need for written informed consent.

\section{Conflict of Interest Statement}

The authors have no conflicts of interest to declare.

\section{Funding Sources}

The study was funded by an ALF research grant at the Skane University Hospital, by a grant from the Skåne Region, as well as grants from the Swedish Research Council and VINNOVA. These are national grants from the Swedish government. The study was also supported by the Swedish Heart-Lung Foundation, a charitable fund-raising organization, as well as the Bundy Academy, a private foundation. There was no industry involvement. Funding organizations had no role in the planning, design, or conduct of the study, collection, analysis, or interpretation of data, or preparation, review, or approval of the manuscript.

\section{Author Contributions}

A.M. and U.E. developed and implemented the methodology and wrote the manuscript; A.K. helped implement the methodology and wrote the manuscript; J.L.F., C.H.S., U.E., M.A., and B.L. helped developing and implementing the methodology and proofread the manuscript; T.S. helped developing the study design. All authors read and approved the finally submitted manuscript.

\section{References}

1 Goodacre S, Cross E, Arnold J, Angelini K, Capewell S, Nicholl J. The health care burden of acute chest pain. Heart. 2005 Feb;91(2): 229-30.

2 Richardson DB. Increase in patient mortality at 10 days associated with emergency department overcrowding. Med J Aust. 2006 Mar; 184(5):213-6.

3 Borna C, Kollberg K, Larsson D, Mokhtari A, Ekelund U. The objective CORE score allows early rule out in acute chest pain patients Scand Cardiovasc J. 2018 Dec;52(6):308-14.

4 Fanaroff AC, Rymer JA, Goldstein SA, Simel DL, Newby LK. Does This Patient With Chest Pain Have Acute Coronary Syndrome? The Rational Clinical Examination Systematic Review. JAMA. 2015 Nov;314(18):1955-65.

5 Mokhtari A, Borna C, Gilje P, Tydén P, Lindahl B, Nilsson HJ, et al. A 1-h Combination Algorithm Allows Fast Rule-Out and Rule-In of Major Adverse Cardiac Events. J Am Coll Cardiol. 2016 Apr;67(13):1531-40.

6 Mokhtari A, Lindahl B, Smith JG, Holzmann MJ, Khoshnood A, Ekelund U. Diagnostic Accuracy of High-Sensitivity Cardiac Troponin $\mathrm{T}$ at Presentation Combined With History and ECG for Ruling Out Major Adverse Cardiac Events. Ann Emerg Med. 2016 Dec; 68(6):649-658.e3

7 Neumann JT, Twerenbold R, Ojeda F, Sörensen NA, Chapman AR, Shah AS, et al. Application of High-Sensitivity Troponin in Suspected Myocardial Infarction. N Engl J Med. 2019 Jun;380(26):2529-40.

8 Roffi M, Patrono C, Collet JP, Mueller C, Valgimigli M, Andreotti F, et al. 2015 ESC Guidelines for the management of acute coronary syndromes in patients presenting without persistent ST-segment elevation: Task Force for the Management of Acute Coronary Syndromes in Patients Presenting without Persistent ST-Segment Elevation of the European
Society of Cardiology (ESC). Eur Heart J. 2016 Jan 14;37(3):267-315.

9 Foy AJ, Liu G, Davidson WR Jr, Sciamanna C, Leslie DL. Comparative effectiveness of diagnostic testing strategies in emergency department patients with chest pain: an analysis of downstream testing, interventions, and outcomes. JAMA Intern Med. 2015 Mar;175(3): 428-36.

10 Amsterdam EA, Kirk JD, Bluemke DA, Diercks D, Farkouh ME, Garvey JL, et al.; American Heart Association Exercise, Cardiac Rehabilitation, and Prevention Committee of the Council on Clinical Cardiology, Council on Cardiovascular Nursing, and Interdisciplinary Council on Quality of Care and Outcomes Research. Testing of low-risk patients presenting to the emergency department with chest pain: a scientific statement from the American Heart Association. Circulation. 2010 Oct;122(17):1756-76.

11 Hermann LK, Weingart SD, Duvall WL, Henzlova MJ. The limited utility of routine cardiac stress testing in emergency department chest pain patients younger than 40 years. Ann Emerg Med. 2009 Jul;54(1):12-6.

12 Weinstock MB, Weingart S, Orth F, VanFossen D, Kaide C, Anderson J, et al. Risk for Clinically Relevant Adverse Cardiac Events in Patients With Chest Pain at Hospital Admission. JAMA Intern Med. 2015 Jul;175(7): 1207-12.

13 Chew DP, Lambrakis K, Blyth A, Seshadri A, Edmonds MJ, Briffa T, et al. A Randomized Trial of a 1-Hour Troponin T Protocol in Suspected Acute Coronary Syndromes: The Rapid Assessment of Possible Acute Coronary Syndrome in the Emergency Department With High-Sensitivity Troponin T Study (RAPIDTnT). Circulation. 2019 Nov;140(19):1543-56. 14 Ljung L, Lindahl B, Eggers KM, Frick M, Linder R, Löfmark HB, et al. A Rule-Out Strat- egy Based on High-Sensitivity Troponin and HEART Score Reduces Hospital Admissions. Ann Emerg Med. 2019 May;73(5):491-9.

15 Twerenbold R, Costabel JP, Nestelberger T, Campos R, Wussler D, Arbucci R, et al. Outcome of Applying the ESC 0/1-hour Algorithm in Patients With Suspected Myocardial Infarction. J Am Coll Cardiol. 2019 Jul;74(4): 483-94.

16 Stoyanov KM, Hund H, Biener M, Gandowitz J, Riedle C, Löhr J, et al. RAPID-CPU: a prospective study on implementation of the ESC 0/1-hour algorithm and safety of discharge after rule-out of myocardial infarction. Eur Heart J Acute Cardiovasc Care. 2020 Feb;9(1): 39-51.

17 Mokhtari A, Lindahl B, Schiopu A, Yndigegn T, Khoshnood A, Gilje P, et al. A 0-Hour/ 1-Hour Protocol for Safe, Early Discharge of Chest Pain Patients. Acad Emerg Med. 2017 Aug;24(8):983-92.

18 Tan WC, Inoue K, AbdelWareth L, Giannitsis E, Kasim S, Shiozaki M, et al. The Asia-Pacific Society of Cardiology (APSC) Expert Committee Consensus Recommendations for Assessment of Suspected Acute Coronary Syndrome Using High-Sensitivity Cardiac Troponin $\mathrm{T}$ in the Emergency Department. Circ J. 2020 Jan;84(2):136-43.

19 Jaffe AS. TRAPID or Trapped? Ann Emerg Med. 2016 Jul;68(1):88-91.

20 Hamm CW, Bassand JP, Agewall S, Bax J, Boersma E, Bueno H, et al; ESC Committee for Practice Guidelines. ESC Guidelines for the management of acute coronary syndromes in patients presenting without persistent ST-segment elevation: the Task Force for the management of acute coronary syndromes (ACS) in patients presenting without persistent ST-segment elevation of the European Society of Cardiology (ESC). Eur Heart J. 2011 Dec;32(23):2999-3054. 
21 Khoshnood A, Erlandsson M, Isma N, Yndigegn T, Mokhtari A. Diagnostic accuracy of troponin $\mathrm{T}$ measured $\geq 6 \mathrm{~h}$ after symptom onset for ruling out myocardial infarction. Scand Cardiovasc J. 2020 Jun;54(3):153-61.

22 Jernberg T, Attebring MF, Hambraeus K, Ivert T, James S, Jeppsson A, et al. The Swedish Web-system for enhancement and development of evidence-based care in heart disease evaluated according to recommended therapies (SWEDEHEART). Heart. 2010 Oct; 96(20):1617-21.

23 Ludvigsson JF, Andersson E, Ekbom A, Feychting M, Kim JL, Reuterwall C, et al. External review and validation of the Swedish national inpatient register. BMC Public Health. 2011 Jun;11(1):450.

24 Thygesen K, Alpert JS, Jaffe AS, Simoons ML, Chaitman BR, White HD, et al; Writing Group on the Joint ESC/ACCF/AHA/WHF Task Force for the Universal Definition of Myocardial Infarction; ESC Committee for Practice Guidelines (CPG). Third universal definition of myocardial infarction. Eur Heart J. 2012 Oct;33(20):2551-67.

25 Thygesen K, Mair J, Giannitsis E, Mueller C, Lindahl B, Blankenberg S, et al; Study Group on Biomarkers in Cardiology of ESC Working Group on Acute Cardiac Care. How to use high-sensitivity cardiac troponins in acute cardiac care. Eur Heart J. 2012 Sep;33(18): $2252-7$.
26 Cullen L, Than M, Brown AF, Richards M, Parsonage W, Flaws D, et al. Comprehensive standardized data definitions for acute coronary syndrome research in emergency departments in Australasia. Emerg Med Australas. 2010 Feb;22(1):35-55.

27 Ekelund U, Kurland L, Eklund F, Torkki P, Letterstål A, Lindmarker $\mathrm{P}$, et al. Patient throughput times and inflow patterns in Swedish emergency departments. A basis for ANSWER, A National SWedish Emergency Registry. Scand J Trauma Resusc Emerg Med. 2011 Jun;19(1):37.

28 Kline JA, Mitchell AM, Runyon MS, Jones $\mathrm{AE}, \mathrm{Webb} \mathrm{WB}$. Electronic medical record review as a surrogate to telephone follow-up to establish outcome for diagnostic research studies in the emergency department. Acad Emerg Med. 2005 Nov;12(11):1127-33.

29 Mokhtari A, Dryver E, Söderholm M, Ekelund U. Diagnostic values of chest pain history, ECG, troponin and clinical gestalt in patients with chest pain and potential acute coronary syndrome assessed in the emergency department. Springerplus. 2015 May;4(1): 219.

30 Reichlin T, Schindler C, Drexler B, Twerenbold R, Reiter M, Zellweger C, et al. One-hour rule-out and rule-in of acute myocardial infarction using high-sensitivity cardiac troponin T. Arch Intern Med. 2012 Sep;172(16): 1211-8.
31 Pickering JW, Greenslade JH, Cullen L, Flaws $\mathrm{D}$, Parsonage W, George P, et al. Validation of presentation and $3 \mathrm{~h}$ high-sensitivity troponin to rule-in and rule-out acute myocardial infarction. Heart. 2016 Aug;102(16):1270-8.

32 Chapman AR, Anand A, Boeddinghaus J, Ferry AV, Sandeman D, Adamson PD, et al. Comparison of the Efficacy and Safety of Early Rule-Out Pathways for Acute Myocardial Infarction. Circulation. 2017 Apr;135(17): 1586-96

33 Twerenbold R, Jaeger C, Rubini Gimenez M, Wildi K, Reichlin T, Nestelberger T, et al. Impact of high-sensitivity cardiac troponin on use of coronary angiography, cardiac stress testing, and time to discharge in suspected acute myocardial infarction. Eur Heart J. 2016 Nov;37(44):3324-32.

34 Chew DP, Zeitz C, Worthley M, Grantham H, Beltrame J, Arstall M, et al. Randomized Comparison of High-Sensitivity Troponin Reporting in Undifferentiated Chest Pain Assessment. Circ Cardiovasc Qual Outcomes. 2016 Sep;9(5):542-53.

35 Chew DP, Scott IA, Cullen L, French JK, Briffa TG, Tideman PA, et al. National Heart Foundation of Australia and Cardiac Society of Australia and New Zealand: australian clinical guidelines for the management of acute coronary syndromes 2016. Med J Aust. 2016 Aug;205(3):128-33. 